\title{
ORIGINAL
}

\section{FACTORES ASOCIADOS AL USO DE LA VÍA PULMONAR E INTRAVENOSA EN UNA MUESTRA DE CONSUMIDORES DE HEROÍNA EN GRANADA}

\section{Joan Carles March Cerdà, Eugenia Oviedo-Joekes, Manuel Romero Vallecillos y Emilio Sánchez-Cantalejo}

Escuela Andaluza de Salud Pública. Granada.

\section{RESUMEN}

Fundamento: En los últimos años el consumo de heroína en España se ha estabilizado y el uso de la vía inyectada ha disminuido. No obstante existen importantes diferencias entre comunidades $y$ entre los consumidores por la vía inyectada. El objetivo del presente estudio es conocer vías de administración de heroína en una muestra intencional de usuarios en situación de exclusión social y algunas de las variables que se asocian con ellas.

Métodos: La muestra se compone de 285 participantes (hombres $82,8 \%$; edad media 36,06). El estudio se llevó a cabo en la ciudad de Granada entre julio y octubre de 2002. Se realizaron entrevistas estructuradas por trabajadores de calle y personas conocedoras del medio (iguales), utilizando la técnica de «bola de nieve».

Resultados: El 48,4\% de los participantes consumen heroína por vía pulmonar e intravenosa; el 25,6\% la consumen sólo por vía pulmonar; y un 25,6\% sólo intravenosa. Tras un análisis de regresión se observa que las mujeres, los participantes más jóvenes y los que se han iniciado más tarde en el consumo son quienes menos tienden a utilizar la vía intravenosa. De los participantes que se iniciaron con inhalación el 21,5\% actualmente además de inhalar también se inyectan, y el 79,5\% restante se mantiene en la vía por inhalación.

Conclusiones: Las personas en situación de exclusión social utilizan la vía intravenosa en porcentajes superiores al resto de la comunidad andaluza.

Palabras clave: Heroína. Adicción a la heroína. Aislamiento social. Drogadicción.

\begin{abstract}
Factors Related to the Use of Inhaled and Intravenous Heroin, Granada, Spain

Background: Heroin use has stabilized in recent years in Spain, and the use of injected heroin having decreased. Nevertheless, major differences exist among different Autonomous Communities and among injected heroin users profile. This study is aimed at ascertaining the forms of heroin use (how administered) in an intentional sample of users in a situation of social exclusion and some variables related to said forms of use.
\end{abstract}

Methods: The sample was comprised of 285 participants $(82.8 \%$ males; average age 36.06 ). This study was conducted in the city of Granada in July-October 2002. Structured surveys conducted by onthe-street interviewers and individuals having knowledge of the environment («peers») using the «snowball» technique.

Results: A total of $48.4 \%$ of the participants alternate inhaled and intravenous heroin use; $25.6 \%$ using only inhaled and $25.6 \%$ only intravenous. Following a regression analysis, it was found that the females, the youngest users and those having started use at an older age were at less risk of intravenous heroin use. A total of $21.5 \%$ of the participants who started using heron in inhaled form, also currently use both the injected and inhaled forms, the remaining $79.5 \%$ continue to use the inhaled form.

Conclusions: Among individuals in a situation of social exclusion the intravenous form exceeds the estimated percentages in the Autonomous Community of Andalusia.

Key words: Heroin. Heroin addiction. Social isolation. Drug addiction.

Correspondencia:

Eugenia Oviedo Joekes

Escuela Andaluza de Salud Pública

Cuesta del Observatorio 4

Campus Universitario de Cartuja

18080 Granada

Correo electrónico: eugenia.oviedojoekes.easp@juntadeanda-

lucia.es 


\section{INTRODUCCIÓN}

Los indicadores utilizados para estimar el uso de heroína y los problemas asociados, como el control de la oferta, la admisión a tratamiento, las atenciones en urgencias y los fallecimientos, han ido descendiendo desde 1996, lo que indicaría que el consumo de heroína en el Estado Español ha disminuido en los últimos años ${ }^{1,2}$. Además se observa también cómo han ido ganando espacio otras vías de administración junto a la intravenosa, en especial la inhalación, según los informes obtenidos en las admisiones a tratamiento ${ }^{3}$.

Esta vía pulmonar de administración de la heroína, fumada o inhalada en chinos (en español «fumarse un chino», en inglés «chasing the dragon, chinising») consiste en inhalar los vapores que resultan de calentar la heroína en un trozo de papel de aluminio. El origen del uso de heroína por la vía fumada podría situarse en el Hong Kong de la década de 1950, llegando a Europa aproximadamente en la década de $1970^{4}$. En Holanda, por ejemplo, la inhalación se extendió rápidamente y se estima que actualmente entre el 75 y 90 por ciento de los usuarios consumen la heroína principalmente o exclusivamente por ésta vía ${ }^{5}$. En otros países europeos también se ha ido extendiendo esta forma de consumo ${ }^{6}$.

En España el consumo de heroína por vía intravenosa ha ido disminuyendo, extendiéndose el uso de la misma por la vía pulmonar. En el año 1991 entre las personas admitidas a tratamiento por consumo de heroína, la vía intravenosa agrupaba al $74,7 \%$ de los usuarios ${ }^{7}$, en el año 2001 el uso de ésta descendió al 24,2\% prevaleciendo el consumo por inhalación o en forma de cigarrillos, con un $67,4 \%^{1}$. No obstante existen diferencias por Comunidades Autónomas, encontrando porcentajes por encima del 25 por ciento en el uso de la vía intravenosa como principal ruta de administración de heroína en El País Vasco, Cataluña, Balea- res, Aragón, Navarra, Cantabria, y La Rioja, entre las personas tratadas por primera vez por abuso o dependencia a la misma ${ }^{3}$. En Andalucía la proporción de usuarios de heroína por vía intravenosa en el año 2000, según los registros de admisión a tratamiento, fue de un $31,2 \%$, muy similar al porcentaje registrado en 1998. El 68,8\% restante consume heroína fundamentalmente por la vía por vía pulmonar ${ }^{8}$. Aspectos culturales o de disponibilidad del tipo de sustancia en el mercado pueden influir en la elección de la forma de administración de heroína ${ }^{9}$. En los últimos años el tipo de heroína que más se consume en España es la marrón, prevaleciendo el uso de la vía inyectada en los lugares donde predomina el consumo de la blan$\mathrm{ca}^{10}$.

El análisis de la forma de consumo de heroína tiene su principal fundamento en el protagonismo que cobra la vía parenteral en el contagio y propagación de enfermedades infecciosas principalmente el sida y la hepatitis $\mathrm{B}$ y $\mathrm{C}^{11-15}$. El descenso del uso de la vía intravenosa ha ido acompañada de una disminución de la infección por VIH entre los usuarios de heroína ${ }^{16}$. No obstante, el uso de la vía la pulmonar no descarta posibles transiciones hacia la inyectada ${ }^{17}$, asociada ésta última con una pérdida del control de consumo, marginación y mayor tiempo de consumo ${ }^{18}$ Además el uso de heroína por vía pulmonar producirá una mayor incidencia de problemas pulmonares ${ }^{19}$ y una potencial transmisión de patógenos respiratorios ${ }^{20}$. Por último, otro importante punto a destacar es la menor percepción de riesgo de adicción y daño que se atribuye a la vía pulmonar, siendo la vía de inicio del consumo en los usuarios más jóvenes, así como la ruta de administración de heroína preferida por ellos ${ }^{21-23}$.

El objetivo del presente estudio es conocer vías de administración de heroína en una muestra intencional de usuarios en situación de exclusión social y algunas de las variables que se asocian con ellas. 


\section{SUJETOS Y MÉTODO}

El presente estudio responde a un diseño transversal. El trabajo de campo se llevó a cabo en la ciudad de Granada entre los meses de julio y octubre de 2002. En esta área de investigación es harto sabido el difícil acceso que se tiene, en general, a la población de usuarios de heroína. El reclutamiento de participantes para los estudios se realiza bien a través de los recursos sanitarios (por ejemplo los datos brindados por los organismos oficiales), o a través del muestreo dirigido en poblaciones diana combinado con la técnica de bola de nieve. El muestreo dirigido implica el abordaje de puntos geográficos claves, seleccionando in situ a los participantes del estudio. Ésta técnica permite llegar a un sector de drogodependientes que no utiliza los servicios sanitarios, aunque posiblemente siguen siendo los grupos más marginales los que no participan en los estudios y, por lo tanto, los que no están reflejados en ellos. Por ello, en este trabajo se ha empleado una forma de acercamiento a las denominadas poblaciones ocultas, apoyándose en la intervención de personas que por sus características tienen un acceso privilegiado a las mismas. El empleo de personas pertenecientes y representantes de la población diana, por ejemplo ex toxicómanos, ha mostrado ser efectiva para recoger información de usuarios de drogas que no acuden regularmente a los servicios sociales y sanitarios ${ }^{24-28}$, dado que la familiaridad y credibilidad que poseen en el grupo diana facilita el contacto y la colaboración para realizar las entrevistas ${ }^{29}$. Con el fin de garantizar la máxima representatividad y diversidad de la población en estudio se maximizó la dispersión de los puntos de muestreo, resultando un total de 55 enclaves considerados como representativos por la tipología de la población que a ellos acude, usuarios de heroína en situación de exclusión social. Los principales escenarios fueron plazas, «chutaderos», comedores sociales, y otros puntos estratégicos ya sea en zonas marginales o aquellas céntricas donde la población diana recurre a procurar- se dinero con actividades informales como aparcar coches en la vía pública.

La muestra es intencional y se compuso de 285 personas usuarias de heroína y en situación de exclusión social en el momento de realizarse la entrevista. Los participantes fueron entrevistados en su mayoría en la calle, plazas y en sus lugares habituales de reunión del municipio de Granada.

Se utilizó una entrevista estructurada, cara a cara, con un tiempo de administración de 25 minutos. El cuestionario recogía información necesaria para estimar el número de consumidores de heroína por vía endovenosa en situación de exclusión social en la ciudad de Granada. Además de algunos datos demográficos se solicitó información sobre la edad de inicio del consumo, patrones y frecuencia de consumo de heroína en el momento de la entrevista, tratamientos realizados y situación socio-familiar (vivienda, apoyo y juicios pendientes).

Las entrevistas fueron realizadas alternativamente por dos personas, un profesional de la salud y un ex usuario de heroína, ambos conocedores del universo de estudio en la ciudad de Granada. Además del abordaje directo de las personas en sus lugares habituales de reunión, el muestreo se apoyó en la técnica de bola de nieve. Las entrevistas se realizaron en el mismo momento y lugar de contacto con el participante, y no se entregó ninguna retribución por contestarla.

Análisis estadísticos: Un primer abordaje de los datos se realizó a través de los estadísticos descriptivos, lo que nos ha permitido conocer las características básicas de la muestra en estudio. Para analizar la relación entre variables categóricas se utilizó el estadístico chi cuadrado de Pearson. En los análisis sobre diferencias de edad, años de consumo o años de tratamiento, se procedió al contraste de medias. Para examinar las variables que se asocian al uso de heroína inyectada se ha construido un modelo de 
regresión logística binaria por pasos (se inyecta vs no se inyecta), introduciendo aquellas variables que mostraron asociaciones significativas y eliminando del modelo aquellas que no alcanzaron una $\mathrm{p}<0,1$. Los análisis se llevaron a cabo con el paquete estadístico SPSS 11.0 para Windows ${ }^{30}$.

\section{RESULTADOS}

En la tabla 1 se pueden apreciar las características sociodemográficas y del consumo de heroína. La muestra estuvo compuesta por 285 personas, de las cuales el $82,8 \%$ son hombres. La edad media (M) es de 36,06 años, con una desviación típica (DT) igual a 6,09, siendo el mínimo de edad 19 años y 59 el máximo. Un importante grupo de participantes no convive con otras personas, el $11 \%$ no tiene techo y el $26,5 \%$ vive solo. El apoyo ante situaciones difíciles proviene en su mayor parte de los padres $(16,5 \%)$ o la familia en general $(28,8 \%)$, aunque los amigos son también una fuente de apoyo $(16,6 \%)$. No obstante un alto porcentaje de los participantes declara no tener a quien recurrir para resolver sus problemas $(33,7 \%)$. En cuanto a la situación judicial el $37,5 \%$ de los participantes tiene juicios pendientes de resolver.

El 79,9\% de los participantes tiene una frecuencia de consumo de heroína diaria; el resto la consume varias veces en semana. Las vías de consumo de heroína se reparten

Tabla 1

Descriptivos de variables socio-demográficas $(\mathbf{n = 2 8 5})$

\begin{tabular}{|c|c|c|}
\hline & & $\%$ \\
\hline \multirow{2}{*}{ Sexo } & Hombre & 82,8 \\
\hline & Mujer & 17,2 \\
\hline Edad (Media en años; Desviación Típica) & $36,06(6,09)$ & \\
\hline \multirow{5}{*}{ Convivencia } & Sin techo & 11,0 \\
\hline & Vive solo & 26,5 \\
\hline & Con amigos & 10,6 \\
\hline & $\begin{array}{l}\text { Con su familia } \\
\text { propia }\end{array}$ & 22,6 \\
\hline & Con sus padres & 29,3 \\
\hline \multirow{5}{*}{ Recibe apoyo de } & La familia & 28,8 \\
\hline & Los padres & 16,5 \\
\hline & Los amigos & 16,8 \\
\hline & otros & 4,2 \\
\hline & Nadie & 33,7 \\
\hline \multirow{2}{*}{ Juicio pendiente } & $\mathrm{Si}$ & 37,7 \\
\hline & No & 62,3 \\
\hline
\end{tabular}


de la siguiente manera: un 48,4\% consumen alternadamente heroína por vía pulmonar e intravenosa; un 25,6\% consumen sólo heroína por vía pulmonar; y un $25,6 \%$ sólo heroína intravenosa. La edad media en el inicio de consumo de heroína es de 22,44 años, siendo el de consumo por vía pulmonar de 23,59 años, y del consumo de intravenosa de 21,89 años. Quienes exclusivamente inhalan heroína, llevan realizando esta práctica una media de 11,83 años, y quienes sólo utilizan la vía intravenosa 14,39 años. El 69\% de los participantes está actualmente en tratamiento, que casi en su totalidad consiste en programas de mantenimiento con metadona. El $48,2 \%$ ha realizado dos o más tratamientos de este tipo. Además el 43,9\% ha realizado tratamientos psicológicos alguna vez.

En la tabla 2 se pueden apreciar las variables que han mostrado asociaciones estadísticamente significativas con el uso de la vía inyectada. La utilizan en menor proporción las mujeres y quienes no tienen juicios pendientes. En cuanto a la vía de inicio, quiénes no diferencian o se han iniciado con la vía intravenosa, en su totalidad se inyectan actualmente. Entro los que se iniciaron con la vía pulmonar el 21,5\% actualmente también usan la intravenosa. Una mayor proporción de usuario por vía intravenosa la encontramos entre quienes en el pasado han realizado más de dos tratamientos con metadona. En cuando a la edad usuario por vía intravenosa son mayores, se han iniciado más jóvenes en el consumo, y por lo tanto llevan más tiempo consumiendo.

Para analizar los factores que en esta muestra se asocian a la forma de consumo de heroína se llevó a cabo un análisis de regresión logística binaria (tabla 3) utilizando los siguientes niveles de la variable dependiente: se inyecta heroína los que se inyectan e

Tabla 2

Descriptivos de variables sobre el consumo de heroína $(\mathbf{n}=\mathbf{2 8 5})$

\begin{tabular}{|c|c|c|}
\hline & & $\%$ \\
\hline \multirow{3}{*}{ Edad de inicio de consumo de heroína (M; DT) } & General & $22,44(6,43)$ \\
\hline & pulmonar & $23,59(7)$ \\
\hline & Intravenosa & $21,89(5,67)$ \\
\hline \multirow{3}{*}{ Tiempo de consumo de heroína (M; DT) } & General & $13,60(5,68)$ \\
\hline & pulmonar & $11,83(5,77)$ \\
\hline & Intravenosa & $14,39(5,55)$ \\
\hline \multirow{3}{*}{ Vía de Consumo de la Heroína } & ambas & 48,4 \\
\hline & Sólo Intravenosa & 25,8 \\
\hline & Sólo pulmonar & 25,8 \\
\hline \multirow{3}{*}{ Con qué vía se inició en el consumo de heroína } & Intravenosa & 40,6 \\
\hline & pulmonar & 33,5 \\
\hline & No discrimina & 26,0 \\
\hline \multirow{3}{*}{ Consume a diario heroína } & General & 79,9 \\
\hline & pulmonar & 45,7 \\
\hline & Intravenosa & 71,0 \\
\hline \multirow{2}{*}{ Actualmente en tratamiento por drogas } & $\underline{\text { Sí }}$ & 69,0 \\
\hline & No & 31,0 \\
\hline \multirow{2}{*}{ Tipo de tratamiento } & Metadona & 98,0 \\
\hline & Otros & 2,0 \\
\hline \multirow{3}{*}{ Metadona en el pasado } & Nunca & 38,0 \\
\hline & Menos de dos & 13,7 \\
\hline & Más de dos & 48,2 \\
\hline \multirow{2}{*}{ Tratamiento psicológico alguna vez } & $\underline{\mathrm{Si}}$ & 43,9 \\
\hline & No & 56,1 \\
\hline
\end{tabular}

M: Media. DT: Desviación típica. 
Tabla 3

Variables asociadas al uso de heroína inyectada

\begin{tabular}{|cc|c|}
\hline & & $\begin{array}{c}\text { \% Intravenosa } \\
\mathbf{N = 2 1 0}\end{array}$ \\
\hline \multirow{2}{*}{ Sexo $^{\text {a }}$} & Hombre & 79,1 \\
\hline \multirow{2}{*}{ Vía de Inicio en el consumo $^{a}$} & Mujer & 49 \\
\cline { 2 - 3 } & Intravenosa & 100 \\
\cline { 2 - 3 } Metadona en el pasado $^{\mathrm{b}}$ & Inhalada & 21,5 \\
\cline { 2 - 3 } & No diferencia & 100 \\
\cline { 2 - 3 } & Nunca & 68,2 \\
\hline \multirow{2}{*}{ Juicio pendiente $^{\mathrm{b}}$} & Menos de dos & 64,1 \\
\cline { 2 - 3 } & Dos o más & 81,0 \\
\hline & Sí & 81,3 \\
\hline
\end{tabular}

$\mathrm{a}=\mathrm{p}<0,00$

$\mathrm{b}=\mathrm{p}<0,05$

inhalan y los que sólo se inyectan frente a los que no se inyecta (sólo inhaladores). En el modelo se introdujeron aquellas variables que mostraron asociaciones estadísticamente significativas con el uso de la vía inyectada (tabla 2). La prueba de Hosmer y Lemeshow para este modelo no es significativa, indicando que los datos se ajustan al mismo. Cuatro factores se muestran asociados a una mayor probabilidad de inyectarse: género, edad, edad de inicio en el consumo y número de tratamientos anteriores con metadona. Así, a igualdad de años y de edad de inicio en el consumo, el riesgo de inyectarse heroína de los hombres con respecto al de las mujeres es casi 3 veces mayor. Por cada año de edad el riesgo de inyectarse se multiplica por 1,1 ; y por cada año que se retrase el inicio en el consumo de heroína el riesgo de inyectarse disminuye en un $13 \%$ aproximadamente. Quienes han efectuado dos o más tratamientos anteriores con metadona tienden a utilizar la vía inyectada en mayor medida en comparación con quienes no han realizado ninguno.

Por último, se ha analizado la vía actual de consumo de heroína en relación con la vía con la que se inició el consumo. 20 (21,5\%) de los participantes que se iniciaron con la vía pulmonar actualmente además de inhalar también se inyectan. El $79 \%$ restante se mantiene consumiendo exclusivamente por vía pulmonar. No existen diferencias de edad estadísticamente significativas entre estos grupos, como se ve en la figura 1. En cuanto a los usuarios que se iniciaron con la vía intravenosa ninguno de ellos la ha abandonado, aunque algunos la alternen con la vía pulmonar.

\section{DISCUSIÓN}

En esta muestra, formada por personas en situación de exclusión social el consumo de heroína por vía intravenosa supera ampliamente los porcentajes estimados en la comunidad andaluza a partir de los pacientes admitidos a tratamiento ${ }^{8}$. Los factores que mejor explican la probabilidad de inyectarse son el sexo, la edad, la edad de inicio en el consumo y el número de tratamientos con metadona realizados con anterioridad. Las mujeres que han participado en el estudio prefieren la vía pulmonar, lo cual se ha indicado también en otros trabajos $^{31}$. Por otro lado, los más jóvenes y quienes se han iniciado más tardíamente en el consumo de heroína tienden a usar la vía 
Figura 1

\begin{abstract}
Diagrama de cajas para usuarios que se iniciaron en el consumo por la vía inhalada, en razón de la vía actual de consumo de heroína y edad
\end{abstract}

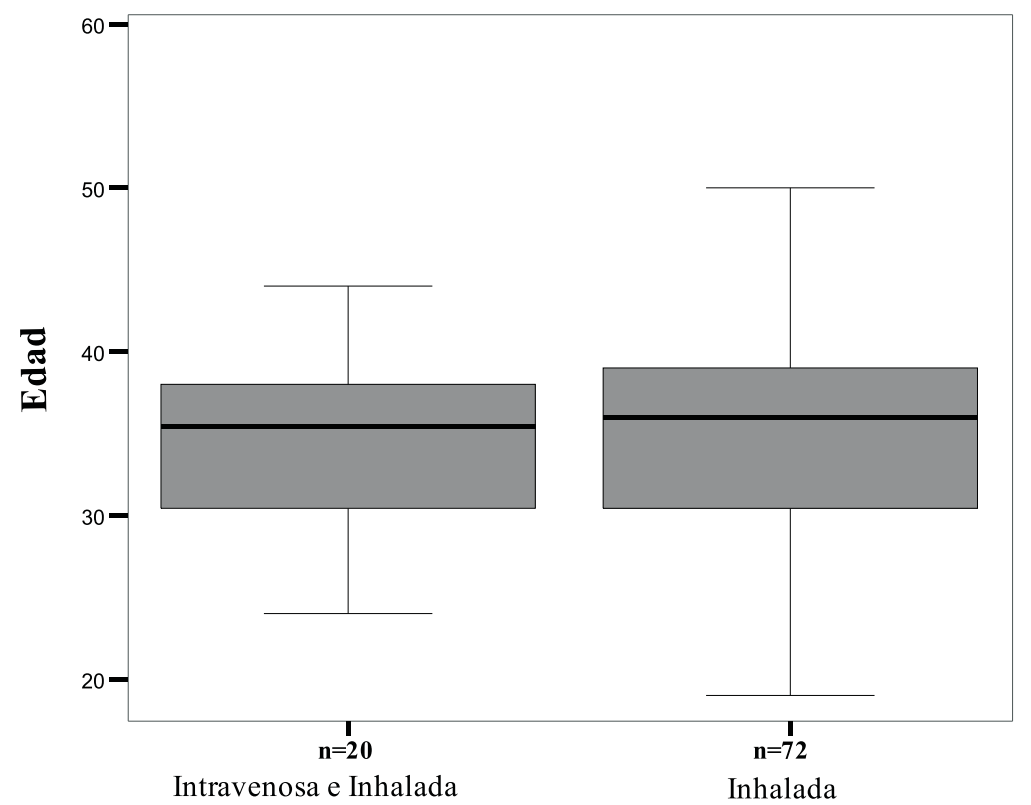

Consumo de Heroína

inyectada en menor medida. La edad de inicio en el consumo, como factor de riesgo, es una constante en los estudios sobre drogas, donde se indica que cuánto más jóvenes comienzan las personas a consumir mayores son los daños asociados ${ }^{32}$. En otros trabajos podemos observar edades tempranas de inicio en el uso de heroína en grupos que consumen preferentemente por la vía intravenosa $^{33}$, así como una mayor inclinación por parte de los usuarios más jóvenes hacia la vía pulmonar ${ }^{34}$. El consumo por la vía pulmonar podría estar acercando la heroína a poblaciones jóvenes ${ }^{35}$, posiblemente por su rápida biodisponibilidad con la ventaja de poder administrársela sin necesidad de utilizar una aguja ${ }^{36-37}$. Por último, quienes han realizado más de dos tratamientos con metadona, en comparación con los que no han realizado ninguno, tienden a usar la vía inyectada en mayor medida. Posiblemente aquellos usuarios de heroína para los cuales los tratamientos disponibles no han sido efectivos sean los que presentan conductas de mayor riesgo asociadas al consumo y, por ende, suman más problemas a su dependencia.

Si bien entre los participantes que se iniciaron en el consumo por la vía pulmonar un $21 \%$ actualmente la alterna con la vía intravenosa, casi el $80 \%$ restante no ha cambiado de vía y esto nos indicaría que quienes se inician en el consumo de heroína inhalada en general continúan con esa forma de consumo. Aunque en ciertos trabajos se ha planteado que la vía pulmonar es una puerta para el consumo de heroína por vía intravenosa ${ }^{17}$, otros establecen que los usuarios mantienen esta vía sin pasarse a la inyectada ${ }^{38}$. En gene- 
Tabla 4

Variables asociadas al uso de heroína inyectada

\begin{tabular}{|cc|c|}
\hline \multicolumn{3}{|c|}{ Contraste de medias } \\
\hline \multicolumn{2}{|c|}{$\begin{array}{c}\text { Intravenosa y } \\
\text { pulmonar }\end{array}$} & $\begin{array}{c}\text { Sólo } \\
\text { pulmonar }\end{array}$ \\
\hline Edad media de la muestra & 36,27 & 35,51 \\
\hline Edad media de inicio en el Consumo $^{\text {a }}$ & 21,55 & 25,00 \\
\hline Tiempo consumiendo heroína $^{\text {a }}$ & 14,72 & 10,42 \\
\hline
\end{tabular}

$\mathrm{a}=\mathrm{p}<0,00$

$\mathrm{b}=\mathrm{p}<0,05$

Tabla 5

Modelo de regresión logística para el consumo de heroína por vía intravenosa

\begin{tabular}{|lcc|}
\hline Variables en el modelo & p & OR (IC. 95\%) \\
\hline Edad & 0,004 & $1,101(1,03-1,18)$ \\
\hline Hombre vs Mujer & 0,006 & $2,187(1,34-5,92)$ \\
\hline Edad de inicio en el consumo & 0,000 & $0,8770(, 83-0,93)$ \\
\hline Tratamientos anteriores con metadona & 0,113 & \\
\hline Nunca & 0,800 & $0,8900(, 36-2,2)$ \\
\hline Menos de dos & 0,071 & $1,833(, 95-3,54)$ \\
\hline Dos o más & & 0,823 \\
\hline $\begin{array}{l}\text { Prueba de bondad de ajuste (Hosmer y } \\
\text { Lemeshow) }\end{array}$ & \\
\hline
\end{tabular}

ral las explicaciones propuestas para el paso hacia del uso de la vía inyectada desde la pulmonar son la presión social, tener una pareja que se inyecta, la influencia del mercado, cambios en las costumbres, entre otras $^{39-40}$. También, se ha sugerido que una mayor frecuencia en el consumo por vía pulmonar, indicador de mayor dependencia, podría estar asociada al cambio hacia la vía inyectada, por considerarse ésta más eficaz y efectiva $^{41}$. En concordancia con otros estudios no se han observado en esta muestra transiciones inversas, es decir, de usuarios por vía intravenosa a la vía pulmonar ${ }^{42}$, dado que ninguno de los participantes que se iniciaron con la vía intravenosa ha dejado de inyectarse, si bien algunos tienden a alternar las vías.

Las consecuencias menos perjudiciales del uso de la vía pulmonar para el consumo de heroína frente a la vía intravenosa, la califican como una ruta de administración de menor riesgo no sólo en lo que al contagio de VIH se refiere ${ }^{43,44}$, sino también con otros aspectos relacionados con la salud, como el deterioro de las venas o infecciones relacionadas con la venopunción. Así mismo, los estudios indican que el consumo de heroína no inyectada conlleva un riesgo menor de sobredosis ${ }^{45,46}$, y puede asociarse a una menor dependencia ${ }^{47}$. 
Los resultados de este trabajo nos muestran que el uso de la vía intravenosa en personas socialmente excluidas excede los porcentajes estimados en la comunidad andaluza y que, en el caso de los usuarios de heroína por vía pulmonar es necesario considerar en la atención sanitaria actividades de prevención de cambio a la inyectada. Algunas alternativas de actuación a este respecto han mostrado ser efectivas, por ejemplo el tratamiento de la dependencia en usuarios de heroína pulmonar, que se comportaría como un factor de protección del paso hacia la vía inyectada ${ }^{48}$. Por otro lado, apoyarse en personas pertenecientes a la población diana, para activamente informar e influir en los hábitos de salud es una estrategia que se ha mostrado efectiva en la prevención de conductas de riesgo de contagio de $\mathrm{VIH} 22^{49}$. Este abordaje de educación llevado a cabo por iguales podría aplicarse también con el objetivo de evitar que los usuarios de drogas por vía pulmonar comiencen a inyectarse o vuelvan a hacerlo.

El presente estudio nos ha permitido acceder a una muestra de consumidores de heroína con un alto porcentaje de participantes que mantienen un contacto irregular o nulo con los servicios socio-sanitarios. El apoyo en personas con acceso privilegiado a la población diana, o «iguales», ha derivado en una mayor colaboración y una mayor tasa de respuesta, permitiéndonos recoger información sobre usuarios de heroína que en general no forman parte de los estudios, y que pasan a constituir las cifras ocultas.

Resulta contradictorio decir que los participantes mantienen un contacto irregular o nulo con los servicios ya que casi el 70 por ciento refiere estar actualmente en tratamiento con metadona. Este resultado debe analizarse teniendo en cuenta el perfil de la muestra, donde muchos de los participantes se consideran usuarios de los programas de metadona, aunque sólo recogen la medicación, a veces ni siquiera ellos mismo (un familiar lo recoge por ellos), muchas veces venden en el mercado ilegal la dosis que recogen y en general consumen varias drogas, incluida la metadona. Éstos pacientes consideran que están en tratamiento con metadona por el simple hecho de tomarla de vez en cuando, no beneficiándose del resto de recursos que éstos programas pueden ofertar.

Este estudio presenta varias limitaciones además de la propias del diseño transversal, principalmente aquellas que se refieren a la generalización de los resultados a otras poblaciones. Cada comunidad tiene sus propios formas y grados de exclusión, generando asociaciones con el uso problemático de heroína con características particulares. Por otra parte, la misma técnica de recogida de datos, el uso de iguales combinado con el de bola de nieve puede haber producido sesgos en el perfil de la muestra, por ejemplo al ser varones los dos entrevistadores la muestra de mujeres podría haber estado subestimada.

\section{BIBLIOGRAFÍA}

1. Observatorio Español sobre Drogas. Informe 6 . Madrid: Delegación del Gobierno para el Plan Nacional sobre Drogas; 2003.

2. European Monitoring Centre for Drugs and Drug Addiction. Spanish National Report 2002. Luxembourg: Office for Official Publications of the European Communities; 2002.

3. Observatorio Español sobre Drogas. Indicadores de Tratamiento, Urgencias y Mortalidad. Informe Ano 2002. Madrid: Delegación del Gobierno para el Plan Nacional sobre Drogas; 2003.

4. Strang J, Griffiths P, Gossop M. Heroin smoking by 'chasing the dragon': origins and history. Addiction 1997; 92(6):673-83; discussion 685-95.

5. van den Brink W, Hendriks VM, Blanken P, Huijsman IA, van Ree JM. Central Committee on the Treatment of Heroin Addicts (CCBH). Medical coprescription of heroin: Two randomized controlled trials. Utrecht: Central Committee on the Treatment of Heroin Addicts; 2002. 
6. Smyth BP, O'Brien M, Barry J. Trends in treated opiate misuse in Dublin: the emergence of chasing the dragon. Addiction 2000; 95(8):1217-23.

7. Observatorio Español sobre Drogas. Informe 3. Madrid: Delegación del Gobierno para el Plan Nacional sobre Drogas., 2000.

8. Junta de Andalucía. Los andaluces ante las drogas VII. Sevilla: Junta de Andalucía, Comisionado para la Droga; 2001.

9. van Ameijden EJ, Coutinho RA. Large decline in injecting drug use in Amsterdam, 1986-1998: explanatory mechanisms and determinants of injecting transitions. J Epidemiol Community Health 2001; 55(5):356-63

10. de la Fuente L, Saavedra P, Barrio G, Royuela L, Vicente J. Temporal and geographic variations in the characteristics of heroin seized in Spain and their relation with the route of administration. Spanish Group for the Study of the Purity of Seized Drugs. Drug Alcohol Depend 1996; 40(3):185-94.

11. Lauer GM, Walker BD. Hepatitis C virus infection. N Engl J Med 2001; 345(1):41-52.

12. Rhodes T, Stimson GV, Crofts N, Ball A, Dehne K, Khodakevich L. Drug injecting, rapid HIV spread, and the 'risk environment': implications for assessment and response. AIDS 1999; 13 Suppl A:S25969.

13. Monga HK, Rodríguez-Barradas MC, Breaux K et al. Hepatitis $\mathrm{C}$ virus infection-related morbidity and mortality among patients with human immunodeficiency virus infection. Clin Infect Dis 2001; 33(2):240-7.

14. March Cerda JC, Ballesta R, Pepsa E. La drogadicción por vía intravenosa, un problema de salud publica en España. Aten Primaria 2001; 28(10):631-3.

15. Rinken S, Vallecillos MR. The evolution of Spanish HIV prevention policy targeted at opiate users: A review. Drug: Education, Prevention and Policy 2002; 9(1):41-6.

16. Torres-Tortosa M, Ruiz Lopez de Tejada MR, Fernandez-Elias $\mathrm{M}$ et al. Cambios en la vía de administración de la heroína y frecuencia de infección por el virus de la inmunodeficiencia humana. Med Clin (Barc) 1995; 104(7):249-52.

17. Strang J, Griffiths P, Powis B, Abbey J, Gossop M How constant is an individual's route of heroin administration? Data from treatment and non-treatment samples. Drug Alcohol Depend 1997; 46(12):115-8.

18. Neaigus A, Miller M, Friedman SR et al. Potential risk factors for the transition to injecting among non- injecting heroin users: a comparison of former injectors and never injectors. Addiction 2001; 96(6):847-60

19. Buster M, Rook L, van Brussel GH, van Ree J, van den Brink W. Chasing the dragon, related to the impaired lung function among heroin users. Drug Alcohol Depend 2002; 68(2):221-8.

20. Perlman DC, Perkins MP, Paone D et al. «Shotgunning» as an illicit drug smoking practice. J Subst Abuse Treat 1997; 14(1):3-9.

21. Barrio G, De La Fuente L, Lew C, Royuela L, Bravo MJ, Torrens M. Differences in severity of heroin dependence by route of administration: the importance of length of heroin use. Drug Alcohol Depend 2001; 63(2):169-77.

22. Svenson G. Directrices Europeas para la educación entre iguales sobre el sida a jóvenes. Bruselas: Comisión europea; 1998.

23. de la Fuente L, Barrio G, Royuela L, Bravo MJ. The transition from injecting to smoking heroin in three Spanish cities. The Spanish Group for the Study of the Route of Heroin Administration. Addiction 1997; 92(12):1749-63.

24. Friedman SR, Flom PL, Kottiri BJ et al. Consistent condom use among drug-using youth in a high HIV-risk neighbourhood. AIDS Care 2002; 14(4):493-507.

25. March JC, Oviedo-Joekes E, Romero M et al. [The experimental drug prescription program in Andalusia (PEPSA): Procedure for recruiting participants]. Gac Sanit 2004; 18(3):245-7.

26. Kuebler D, Hausser D. The Swiss Hidden Population Study: practical and methodological aspects of data collection by privileged access interviewers. Addiction 1997; 92(3):325-34.

27. Griffiths P, Gossop M, Powis B, Strang J. Reaching hidden populations of drug users by privileged access interviewers: methodological and practical issues. Addiction 1993; 88(12):1617-26.

28. Romero M, Zunzunegui MV, Perea E, Gornemann I, Fernandez A. Uso consistente del condón entre los usuarios de droga por vía intravenosa y sus parejas estables. Gac Sanit 1999; 13(2):96-101. 
29. Ouellet LJ, Wiebel WW, Jimenez AD. Team research methods for studying intranasal heroin use and its HIV risks. NIDA Res Monogr 1995; 157:182-211.

30. SPSS for windows SPSS I, Version 11.0. Chicago: 2002.

31. Gossop M, Griffiths P, Strang J. Sex differences in patterns of drug taking behaviour. A study at a London community drug team. Br J Psychiatry 1994; 164(1):101-4

32. Anthony JC, Petronis KR. Early onset drug use and risk of later drug problems. Drug Alcohol Depend 1995; 40(1):9-15.

33. Cravioto P, Medina-Mora ME, de la Rosa B, Galvan F, Tapia-Conyer R. Patrones de consumo de heroína en una cárcel de la frontera norte de México: barreras de acceso a tratamiento. Salud Publica Mex 2003; 45(3):181-90.

34. de la Fuente L, Barrio G, Bravo MJ, Royuela L. Heroin smoking by «chasing the dragon»: its evolution in Spain. Addiction 1998; 93(3):444-6.

35. Gervin M, Hughes R, Bamford L, Smyth BP, Keenan E. Heroin smoking by «chasing the dragon» in young opiate users in Ireland: stability and associations with use to «come down» off «Ecstasy». J Subst Abuse Treat 2001; 20( 4):297-300.

36. Comer SD, Collins ED, MacArthur RB, Fischman MW. Comparison of intravenous and intranasal heroin self-administration by morphine-maintained humans. Psychopharmacology (Berl) 1999; 143(4):327-38.

37. Sotheran J, Goldsmith D, Blasco M, Friedman R. Heroine sniffing as self-regulatin among injecting and non-injecting heroin users. Journal of Drug Issues 29:401-22.

38. Griffiths P, Gossop M, Powis B, Strang J. Transitions in patterns of heroin administration: a study of heroin chasers and heroin injectors. Addiction 1994; 89(3):301-9.

39. Strang J, Griffiths P, Powis B, Gossop M. Heroin chasers and heroin injectors: differences observed in a community sample in London, UK. Am J Addict 1999; 8(2):148-60.

40. van Ameijden EJ, Coutinho RA. Large decline in injecting drug use in Amsterdam, 1986-1998: explanatory mechanisms and determinants of injecting transitions. J Epidemiol Community Health 2001; 55(5):356-63.

41. Bravo MJ, Barrio G, de la Fuente L, Royuela L, Domingo L, Silva T. Reasons for selecting an initial route of heroin administration and for subsequent transitions during a severe HIV epidemic. Addiction 2003; 98(6):749-60.

42. Swift W, Maher L, Sunjic S. Transitions between routes of heroin administration: a study of Caucasian and Indochinese heroin users in south-western Sydney, Australia. Addiction 1999; 94(1):7182

43. de la Fuente L, Bravo MJ, Lew C, Barrio G, Soriano V, Royuela L. Prevalencia de infeccion por el virus de la inmunodeficiencia humana y de conductas de riesgo entre los consumidores de heroina de Barcelona, Madrid y Sevilla: un ejemplo de las ventajas de centrar los estudios en los consumidores y no solo en los usuarios por vía intravenosa. Med Clin (Barc) 1999; 113(17):646-51.

44. Latkin CA, Knowlton AR, Sherman S. Routes of drug administration, differential affiliation, and lifestyle stability among cocaine and opiate users: implications to HIV prevention. J Subst Abuse 2001; 13(1-2):89-102.

45. Darke S, Ross J. Fatal heroin overdoses resulting from non-injecting routes of administration, NSW, Australia, 1992-1996. Addiction 2000; 95(4):56973.

46. Gossop M, Griffiths P, Powis B, Williamson S, Strang J. Frequency of non-fatal heroin overdose: survey of heroin users recruited in non-clinical settings. BMJ 1996; 313(7054):402.

47. Smolka M, Schmidt LG. The influence of heroin dose and route of administration on the severity of the opiate withdrawal syndrome. Addiction 1999; 94(8):1191-8.

48. Kelley MS, Chitwood DD. Effects of drug treatment for heroin sniffers: a protective factor against moving to injection? Soc Sci Med 2004; 58(10):2083-92.

49. Friedman SR, Des Jarlais DC, Sotheran JL, Garber J, Cohen H, Smith D. AIDS and self-organisation among intravenous drug users. The International Journal of the Addictions 1987; 3:201-19. 